\title{
A NEW PROOF OF WEIERSTRASS'S THEOREM CONCERNING THE FACTORIZATION OF A POWER SERIES.
}

BY PROFessor GILBERT AMES BLISS.

THE theorem which is to be proved here may be stated in the following form:

Let $f\left(x_{1}, x_{2}, \ldots, x_{p}, y\right)$ be a convergent series in $x_{1}, x_{2}, \ldots$, $x_{p}, y$, and such that the series $f(0,0, \ldots, 0, y)$ begins with the term of degree $n$. Then $f\left(x_{1}, x_{2}, \cdots, x_{p}, y\right)$ is factorable in the form

$$
\begin{aligned}
f\left(x_{1}, x_{2}, \cdots, x_{p}, y\right)=\left(a_{0}+a_{1} y+a_{2} y^{2}+\cdots\right. \\
\left.+a_{n-1} y^{n-1}+y^{n}\right) \phi\left(x_{1}, x_{2}, \ldots, x_{p}, y\right),
\end{aligned}
$$

where $a_{0}, a_{1}, \ldots, a_{n-1}$ are convergent power series in $x_{1}, x_{2}, \ldots, x_{p}$ which vanish for $x_{1}=x_{2}=\cdots=x_{p}=0$, and $\phi$ is a power series in $x_{1}, x_{2}, \ldots, x_{p}, y$ which has a constant term different from zero.

In the Bulletin de la Société Mathématique de France* Goursat has called attention to the fact that the proof which Weierstrass gave of this important theorem, as well as the later proofs which occur in the literature $\uparrow$ make use of the notions of the function theory, while the theorem itself is essentially of an algebraic character. In the paper referred to he has given an elegant and elementary proof of the theorem which is in outline as follows:

By means of the substitution

$$
y^{n}=-a_{0}-a_{1} y-a_{2} y^{2}-\cdots-a_{n-1} y^{n-1}
$$

the series $f$ can be reduced to a polynomial $P$ of degree $n-1$ in $y$, whose $n$ coefficients are convergent series in $a_{0}, a_{1}, \ldots$, $a_{n-1}, x_{1}, x_{2}, \cdots, x_{p}$. By the usual theorems in implicit function theory it is shown that the $n$ equations found by putting these coefficients equal to zero have unique solutions for $a_{0}, a_{1}, \ldots$, $a_{n-1}$ as power series in $x_{1}, x_{2}, \cdots, x_{p}$ which vanish with $x_{1}, x_{2}$,

\footnotetext{
* "Démonstration élémentaire d'un théorème de Weierstrass," vol. 36 (1908), p. 209.

$\dagger$ Picard, Traite d'Analyse, vol. II, p. 243 ; Goursat, Cours d'Analyse, vol. II, p. 284 .
} 
$\cdots, x_{p}$. If the values so found are substituted in the formula

$$
y^{n}=-a_{0}-a_{1} y-a_{2} y^{2}-\cdots-a_{n-1} y^{n-1}+\mu
$$

and the series $f$ again reduced, a polynomial $P_{1}$ of degree $n-1$ in $y$ will be found whose coefficients are series in $x_{1}, x_{2}, \cdots, x_{p}, \mu$. On account of the way in which the functions $a_{0}, a_{1}, \ldots, a_{n-1}$ were determined, this polynomial $P_{1}$ has a factor $\mu$ and hence $f$ has a factor $\left(a_{0}+a_{1} y+\cdots+a_{n-1} y^{n-1}+y^{n}\right)$.

The proof which is given below seems to the writer even more direct than that of Goursat, and it furnishes besides convenient formulas for the determination of the coefficients of the series $a_{0}, a_{1}, \ldots, a_{n-1}$.

The series $f$ can evidently be written in the form

$$
f=-y^{n}+f_{0}+f_{1} y+f_{2} y^{2}+\cdots+f_{n} y^{n}+\cdots,
$$

where the coefficients $f_{l}$ are power series in $x_{1}, x_{2}, \ldots, x_{p}$, and $f_{1}, f_{2}, \cdots, f_{n}$ have no constant terms. If a convergent series

$$
b=b_{0}+b_{1} y+b_{2} y^{2}+\cdots
$$

having its constant term different from zero, together with $n$ other convergent series $\mu_{0}, \mu_{1}, \ldots, \mu_{n-1}$ in $x_{1}, x_{2}, \ldots, x_{p}$, can be determined so that the identity

(2) $b\left(-y^{n}+f_{0}+f_{1} y+\cdots\right) \equiv \mu_{0}+\mu_{1} y+\cdots+\mu_{n-1} y^{n-1}-y^{n}$

is true, then the series $\phi$ of the theorem will be $1 / b$ and the theorem will be proved. By comparison of the coefficients of the different powers of $y$ in (2), the equations

$$
\begin{aligned}
& \mu_{0}=b_{0} f_{0} \\
& \mu_{1}=b_{0} f_{1}+b_{1} f_{0},
\end{aligned}
$$$$
\mu_{n-1}=b_{0} f_{n-1}+b_{1} f_{n-2}+\cdots+b_{n-1} f_{0} \text {, }
$$$$
b_{0}-1=b_{0} f_{n}+b_{1} f_{n-1}+\cdots+b_{n-1} f_{1}+b_{n} f_{0},
$$$$
b_{k}=b_{0} f_{n+k}+b_{1} f_{n+k-1}+\cdots+b_{k-1} f_{n+1}+b_{k} f_{n}+\cdots+b_{n+k} f_{0},
$$ 
are found. These equations determine uniquely the coefficients of the series $b_{0}, b_{1}, \cdots ; \mu_{0}, \mu_{1}, \ldots, \mu_{n-1}$ as rational integral functions with positive coefficients of the coefficients of the series $f_{0}, f_{1}, f_{2}, \ldots$. For on account of the fact that $f_{0}, f_{1}, \ldots, f_{n}$ have no constant terms, the terms of order $m$ in $b_{k}$ can be determined from the last equation in the form just described as soon as the terms of order $m$ and less in $b_{0}, b_{1}, \ldots, b_{k-1}$, and those of order $m-1$ and less in $b_{k}, b_{k+1}, \ldots, b_{k+n}$, are known. Suppose, for example, that the terms of order zero of all the $b$ 's up to and including $b_{k+m n}$ have been computed. From them the terms of order one of $b_{0}, b_{1}, \cdots, b_{k+(m-1) n}$ can be found; then the terms of order two of $b_{0}, b_{1}, \ldots, b_{k+(m-2) n}$; and so on, until the terms of order $m$ of $b_{0}, b_{1}, \ldots, b_{k}$ are obtained. Hence step by step the terms of the different orders can be determined for all of the series $b_{k}$, and hence for all the series $\mu$. It is evident, therefore, that if there exist convergent series $b, \mu_{0}$, $\mu_{1}, \cdots, \mu_{n-1}$ satisfying identically the relation (2), then those series have coefficients which are uniquely determined by the relations (3). Furthermore if a function $F$ of the form (1) can be found for which the coefficients in $F_{0}, F_{1}, \ldots$ are positive and greater in numerical value, respectively, than those of $f$, and such that the corresponding series $B, M_{0}, M_{1}, \ldots, M_{n-1}$ for $F$ are convergent, then the series $b, \mu_{0}, \mu_{1}, \cdots, \mu_{n-1}$ for $f$ will also be convergent.

A function $F$ of the type desired can readily be found. The series $f$ can be supposed without loss of generality to be convergent for $x_{1}=x_{2}=\cdots=x_{p}=y=1$. For if it were convergent for $\left|x_{i}\right| \leqq \rho_{i},|y| \leqq \rho$, it would only be necessary to make the transformation $x_{i}=\rho_{i} x_{i}^{\prime}, y=\rho y^{\prime}$ in order to have a series with the desired property. If the values $x_{1}=x_{2}=\cdots=$ $x_{p}=y=1$ are substituted in $f$, the resulting series is the series of the coefficients of $f$ and is absolutely convergent. Hence each coefficient of $f$ is in absolute value less than a certain positive constant $N$. For the function $F$, then, let

$$
\begin{gathered}
F_{0}=F_{1}=\cdots=F_{n}=N\left[\frac{1}{\left(1-x_{1}\right)\left(1-x_{2}\right) \cdots\left(1-x_{p}\right)}-1\right], \\
F_{n+k}=N \frac{1}{\left(1-x_{1}\right)\left(1-x_{2}\right) \cdots\left(1-x_{p}\right)},
\end{gathered}
$$

where $k=1,2, \ldots, \infty$. Every coefficient of $F$ is positive and greater in absolute value than the corresponding coefficient of $f$. 
For the function $F$ the relation (2) after a simple transformation takes the form

$$
\begin{aligned}
\frac{B}{1-y}\left[-y^{n}+\frac{N}{\left(1-x_{1}\right)\left(1-x_{2}\right) \cdots\left(1-x_{p}\right)}-N\right. \\
\left.\quad+(1+N) y^{n+1}\right] \equiv M_{0}+M_{1} y+\cdots+M_{n-1} y^{n-1}-y^{n} .
\end{aligned}
$$

Series $B, M_{0}, M_{1}, \ldots, M_{n-1}$ of the type desired can be found satisfying this identity provided that series $C, M_{0}, M_{1}, \ldots, M_{n-1}$ of similar type can be found satisfying the identity

$$
-y^{n}+F_{0}+c y^{n+1} \equiv\left(M_{0}+M_{1} y+\cdots+M_{n-1} y^{n-1}-y^{n}\right) C,
$$

where $c=1+N$. The latter, however, can be satisfied by a linear function $C=M-c y$. For by comparing the coefficients of $y^{n}$ it is found that $M=1-c M_{n-1}$, and by comparing the coefficients of the lower powers

$$
\begin{aligned}
M_{0} & =c M_{0} M_{n-1}+F_{0}, \\
-c M_{0}+M_{1} & =c M_{1} M_{n-1}, \\
-c M_{1}+M_{2} & =c M_{2} M_{n-1}, \\
\cdot \cdot \cdot \cdot \cdot & \cdot \cdot \cdot \cdot \\
-c M_{n-2}+M_{n-1} & =c M_{n-1} M_{n-1} .
\end{aligned}
$$

From the well known theorems concerning implicit functions it follows that these equations have solutions $M_{0}, M_{1}, \ldots, M_{n-1}$ which are power series in $x_{1}, x_{2}, \cdots, x_{p}$, vanishing when $x_{1}=x_{2}$ $=\cdots=x_{p}=0$. Hence the theorem is proved.

It should be noted that the dominant function $F$ and the implicit function theory have been used only in the proof of the convergence of the series $b$ and $\mu_{1}, \mu_{2}, \ldots, \mu_{n-1}$. The computation of the coefficients of these series is effected by means of the equations (3).

The University of Chicago, November, 1909. 